\title{
Psychosocial Factors Associated with Patterns of Smoking Surrounding Pregnancy in Fragile Families
}

\author{
Robin L. Page • Yolanda C. Padilla • \\ Erin R. Hamilton
}

Published online: 1 January 2011

(c) The Author(s) 2010. This article is published with open access at Springerlink.com

\begin{abstract}
Although research has documented factors associated with maternal smoking, we need a more in-depth understanding of the risk factors associated with changes in smoking behaviors during the postpartum period. We investigate smoking patterns during pregnancy and 1 year postpartum as a function of relevant psychosocial factors. We use data on 3,522 postpartum mothers from the Fragile Families and Child Wellbeing Study to analyze the predictors of smoking among mothers who did not smoke during pregnancy but smoked at 1 year postpartum, mothers who smoked both during pregnancy and postpartum, and mothers who did not smoke during either period. Our covariates are grouped into four categories of risk factors for smoking: socioeconomic status, health care, life course and health, and partner and social support. Postpartum mothers in our sample were more likely to smoke throughout or after their pregnancies if they had only a high school education or less, had a household income three or more times below the poverty line, had public or no health insurance, breastfed for less than 5 months, were not married to the infant's father, if the infant's father currently smoked, and if they attended religious services less than once a week. Mental health
\end{abstract}

\author{
R. L. Page ( $\square)$ \\ School of Nursing, University of Texas at Austin, \\ Austin, TX, USA \\ e-mail: rpage@nursing.utexas.edu \\ Y. C. Padilla \\ School of Social Work, University of Texas at Austin, \\ Austin, TX, USA \\ e-mail: ypadilla@austin.utexas.edu \\ E. R. Hamilton \\ Department of Sociology, University of California, Davis, \\ Davis, CA, USA \\ e-mail: erhamilton@ucdavis.edu
}

problems were consistently associated with an increased risk of constant and postpartum smoking relative to nonsmoking. Psychosocial factors play a role in postpartum smoking, but they have a stronger effect in predicting smoking that persists throughout pregnancy and the first year postpartum.

Keywords Postpartum · Pregnancy · Smoking · Psychosocial · Unmarried · Hispanic · Fragile Families

\section{Introduction}

Much attention has been given to the health and health behaviors of women during pregnancy because of direct health risks to the infant. From a research perspective, there has been less of an emphasis on mothers' health and health behaviors during the period following the birth despite the fact that experts view the postpartum period as a "life redefining transition" marked by profound physiologic changes in conjunction with new stressors, such as parenting challenges, body-image changes, and time and role conflicts [1]. As a result, during the postpartum period the mother may focus intently on her baby's well-being while neglecting her own well-being. Behaviors such as alcohol and tobacco use suspended during pregnancy may reemerge in the postpartum period [2]. Of particular interest are maternal health behaviors during pregnancy and postpartum that may not only harm the mother's health but also have potentially negative effects on the health of the child. Exposure to secondhand smoke after birth puts infants at risk. Recent studies show increased emergency room visits, hospitalizations, and sick days due to respiratory illnesses among children ages $0-4$ years who are exposed to secondhand smoke [3]. 
Research on the deleterious effects of cigarette, alcohol, and drug use during pregnancy on neonatal outcomes is widely published [4-7]. Perhaps as a result of the widespread discussion of the ill-effects on infant outcomes, many women abstain from or decrease substance use during pregnancy [8]. Data from the 2004 National Survey on Drug Use and Health [9] show significantly lower rates of tobacco use among pregnant women. Cigarette smoking in the past month was reported by $18 \%$ of pregnant women versus $30 \%$ of nonpregnant women ages 15-44. Similarly, in a study on lay advice regarding alcohol and tobacco use with 105 low-income pregnant women who smoked prior to pregnancy, over half abstained from smoking one or more months during their pregnancies [8].

If women abstain from or quit smoking during pregnancy, an important maternal health question is whether they resume (or begin) smoking after the birth of the child. In a study on smoking relapse in postpartum women, researchers found that $94 \%$ of women who quit smoking during pregnancy reported that concern for the health of their unborn baby was their primary reason for quitting. While $91 \%$ stated that they intended to abstain from smoking after the baby was born, by 6 months postpartum only $30 \%$ of them were still non-smokers [10].

Past studies on smoking during the time surrounding pregnancy suggest that lower income, less education, White ethnicity, age 20-29 years, and not being married to the biological father of the baby place mothers at risk [11]. Contextual factors that also play a role are alcohol consumption, not breastfeeding, number of household smokers, and maternal depression. In a study by Martin et al. [11], women who drank one or more alcoholic drinks per week were more likely to smoke, less likely to quit, and more likely to relapse than women who drank less than that. Women who lived with another smoker were also more likely to smoke at 24 months postpartum. Mothers who did not breastfeed were more than twice as likely to smoke and greater than four times more likely to relapse. Postpartum depression was also positively correlated with smoking at 24 months postpartum but was not related to smoking relapse.

Although research has documented factors associated with maternal smoking, we need a more in-depth understanding of changes in smoking behaviors through the pregnancy and postpartum periods. Our study adds to the existing literature by including more in-depth measures of maternal psychosocial factors that may contribute to postpartum smoking. We examine whether the risk profile for smoking during pregnancy is similar to the risk profile for women who abstain from smoking during pregnancy but resume afterwards, or if it is possible to differentiate these postpartum smokers. A psychosocial framework incorporates an individual's psychological well-being, family environment, and access to health and financial resources. We include measures of the mother's mental health, availability of social support, and relationship status with her partner. We also include a religious attendance variable as one measure of access to social support. Greater frequency of religious service attendance has been associated with lower rates of smoking [12, 13]. We differentiate between US-born and foreign-born Mexican origin women in addition to the more commonly analyzed categories of White, Black, and Hispanic women, as research has shown that US-born and foreign-born women differ greatly in their smoking behavior [14]. Information on mother's health insurance, education, and poverty is included. The addition of these variables to the existing literature on smoking among postpartum women can further our understanding of the psychosocial context that may predict which women are most likely to smoke during the vulnerable postpartum phase.

In this study we take a closer look at changes in smoking patterns among women during pregnancy and through the first year postpartum period. We focus on the effects of psychosocial factors among at-risk mothers by observing a sample with a large number of unmarried urban mothers. Unmarried mothers are especially vulnerable after the birth of a child due to the precarious conditions often associated with single parenthood. We explore what contributes to postpartum smoking, that is, smoking after birth, both among women who did not smoke during pregnancy and women who did. Given that many of these postpartum smokers stopped smoking during pregnancy because of the health risks for the infant, we explore what risk factors contribute to their starting smoking again with an eye towards better informing postpartum maternal and child health care.

\section{Methods}

Data

The data used in this study are drawn from the Fragile Families and Child Wellbeing Study conducted by Princeton and Columbia Universities [15]. The Fragile Families Study is an ongoing, longitudinal, birth cohort study of children born to predominantly unmarried parents. The sample was drawn between 1998 and 2000 from hospitals in cities with over 200,000 people and included 4,898 births. Parents were interviewed at the time of the child's birth, as well as at 1,3 , and 5 years following the birth. For this study we draw on data from the birth and 1-year follow-up interviews.

Our analytic sample consists of 3,522 1-year postpartum mothers. Of the 4,898 mothers interviewed at the time of 
the child's birth in the initial baseline sample, we excluded $534(11 \%)$ who were not interviewed at year 1 . We also excluded the 578 mothers who were pregnant at year 1 because their smoking behavior is no longer simply postpartum, 193 mothers in the "other" race category because this is an ambiguous and heterogeneous group, and 71 mothers who smoked during pregnancy but not in the year after birth, a group too small to analyze separately. We used Stata's multiple imputation methods to impute values for 169 mothers who were missing data on one or more analysis variables. Results using the multiple imputation technique did not substantively differ from results using listwise deletion (results available upon request).

\section{Variables}

Our dependent variable is a three-category indicator of pregnancy and postpartum smoking based on maternal self reports. We categorize mothers as "non-smokers," "constant smokers," and "postpartum smokers" based on questions asked about smoking at birth and at the 1-year follow up. At birth, mothers were asked, "During your pregnancy, how many cigarettes did you smoke?" We coded mothers as non-smokers during pregnancy if they answered "none," whereas mothers are coded as smokers during pregnancy if they answered "less than 1 pack a day," "1 or more but less than 2 packs a day," or " 2 or more packs a day." At year one, mothers were asked whether they smoked cigarettes in the past month. We coded mothers as non-smokers in the year after birth if they answered "no" to this question; mothers were coded as smokers in the year after birth if they answered "yes" to this question. Thus, our three smoking categories are (a) non-smokers, mothers who reported that they did not smoke during pregnancy nor during the year following birth, (b) constant smokers, mothers who reported smoking both during pregnancy and in the year following birth; and (c) postpartum smokers, mothers who reported that they did not smoke during pregnancy but did smoke in the year following birth. Table 1 shows the distribution of our sample into these three categories: $72 \%$ of mothers were non-smokers, $17 \%$ were constant smokers, and $10 \%$ were postpartum smokers.

Our covariates are grouped into four categories comprising mothers' psychosocial factors: socioeconomic status, health care, life course and health, and partner and social support. Socioeconomic status variables include mother's race/ethnicity, education, and household poverty status. Based on mothers' self-reports of their race, ethnicity, and nativity at baseline, we categorized mothers as US-born Mexican-origin, foreign-born Mexican-origin, non-Hispanic black, non-Hispanic white, and other Hispanic. Mother's education is categorized as less than high school, a high school degree or its equivalent or some college or higher. Household income-to-poverty ratios were constructed by comparing the mother's reported annual household income to federal poverty guidelines that vary according to household composition (number of children and adults). We distinguish between households below the federal poverty level $(\$ 17,050$ for a family of four in 2000), households with incomes between 100 and $300 \%$ percent of the federal poverty level, and households with incomes greater than $300 \%$ of the federal poverty level.

We include two measures of access to and use of health care, which were taken from the baseline survey. The first is the mother's health insurance coverage, a bivariate indicator distinguishing between private coverage and public or no health insurance coverage. We group public and no health insurance coverage together because very few mothers in our sample reported no health insurance, most likely because those who were uninsured were enrolled in Medicaid during pregnancy or at the time of labor. The second is the trimester when the mother began prenatal care, which distinguishes between the first trimester or other (including no prenatal care).

Life course and health variables include mother's age, parity, mental health problem, parenting stress, and breastfeeding. Mother's age at the time of the child's birth is coded as a dummy variable to indicate mothers who are less than 25 years old. We incorporate a measure of parenting stress that identifies mothers who report that they "strongly agree" that they feel trapped by their responsibilities as a parent, find that taking care of their children is much more work than pleasure, or often feel tired, worn out, or exhausted from raising a family. We also include an indicator to identify mothers who report that they were diagnosed with or prescribed medicine for a mental health problem within the last year. Finally, we include an indicator of whether mothers breastfed for at least 5 months.

Partner and social support measures capture the level of support from the father as well as support from other sources. The first is the mother's relationship status with the baby's father at baseline, which distinguishes between married, cohabiting, dating, and other status (including friends but not romantic, hardly talk, and unknown fathers) parents. We also include a dummy for whether the father suggested an abortion, based on mothers' reports, which may capture the father's support of the mother and the child [16]. A third indicator of social support is the father's status as a smoker. We use data from father reports at baseline and year one about their smoking status. Fathers are defined as smokers if they report smoking at baseline or year one. Because a substantial portion ( $24 \%$ of our sample) of fathers was not interviewed, we also include a dummy variable to indicate whether the father data are 
Table 1 Percent distributions and cross-tabulations of smoking status

\begin{tabular}{|c|c|c|c|c|}
\hline & \multirow{2}{*}{$\begin{array}{l}\text { Percent distribution of } \\
\text { characteristics for full } \\
\text { sample of mothers }\end{array}$} & \multicolumn{3}{|c|}{ Cross-tabulations of smoking status by maternal characteristics (rows total to $100 \%$ ) } \\
\hline & & Non-smoker & Constant smoker & Postpartum smoker \\
\hline All mothers & 100.0 & 72.4 & 17.2 & 10.4 \\
\hline \multicolumn{5}{|l|}{ Socioeconomic status } \\
\hline \multicolumn{5}{|l|}{ Mother's race/ethnicity $* * *$} \\
\hline Non-Hispanic White & 23.2 & 62.4 & 25.4 & 12.1 \\
\hline US-born Mexican & 8.5 & 75.7 & 9.9 & 14.4 \\
\hline Foreign-born Mexican & 7.8 & 94.5 & 1.5 & 4.0 \\
\hline Non-Hispanic Black & 48.5 & 72.6 & 18.5 & 8.9 \\
\hline Other Hispanic & 12.1 & 74.3 & 11.1 & 14.6 \\
\hline \multicolumn{5}{|l|}{ Mother's education*** } \\
\hline Less than high school & 32.6 & 64.1 & 23.2 & 12.6 \\
\hline High school & 30.9 & 70.3 & 19.1 & 10.5 \\
\hline Some college or higher & 36.4 & 81.7 & 9.9 & 8.3 \\
\hline \multicolumn{5}{|l|}{ Poverty*** } \\
\hline$<100 \%$ poverty & 34.2 & 67.5 & 21.8 & 10.7 \\
\hline $100-300 \%$ & 42.0 & 70.9 & 17.4 & 11.7 \\
\hline $300 \%+$ & 23.9 & 82.2 & 10.2 & 7.6 \\
\hline \multicolumn{5}{|l|}{ Health care } \\
\hline \multicolumn{5}{|l|}{ Prenatal care*** } \\
\hline First trimester & 82.7 & 74.4 & 15.6 & 10.0 \\
\hline Late/none & 17.3 & 63.0 & 24.5 & 12.5 \\
\hline \multicolumn{5}{|c|}{ Health insurance coverage * $^{* *}$} \\
\hline Private & 34.1 & 80.9 & 10.6 & 8.5 \\
\hline Public or none & 65.9 & 68.0 & 20.6 & 11.4 \\
\hline \multicolumn{5}{|l|}{ Life course and health } \\
\hline \multicolumn{5}{|l|}{ Mother's age $* * *$} \\
\hline$<25$ & 47.0 & 69.4 & 17.4 & 13.2 \\
\hline$\geq 25$ & 53.0 & 75.8 & 16.9 & 7.3 \\
\hline \multicolumn{5}{|l|}{ Parity*** } \\
\hline First birth & 37.8 & 74.1 & 13.7 & 12.2 \\
\hline Second or higher birth & 62.2 & 71.4 & 19.3 & 9.3 \\
\hline \multicolumn{5}{|l|}{ Mental health problem*** } \\
\hline Yes & 26.8 & 63.7 & 23.7 & 12.7 \\
\hline No & 73.2 & 75.6 & 14.8 & 9.6 \\
\hline \multicolumn{5}{|l|}{ Parenting stress $* *$} \\
\hline Yes & 23.1 & 67.6 & 21.3 & 11.1 \\
\hline No & 76.9 & 73.9 & 15.9 & 10.2 \\
\hline \multicolumn{5}{|c|}{ Breastfed at least 5 months*** } \\
\hline Yes & 23.1 & 85.4 & 6.9 & 7.7 \\
\hline No & 76.9 & 68.5 & 20.2 & 11.2 \\
\hline \multicolumn{5}{|l|}{ Partner and social support } \\
\hline \multicolumn{5}{|c|}{ Parent's relationship status $* * *$} \\
\hline Married & 24.8 & 86.3 & 6.1 & 7.6 \\
\hline Cohabiting & 35.9 & 67.7 & 20.2 & 12.0 \\
\hline Visiting & 26.4 & 68.2 & 20.1 & 11.7 \\
\hline Friends/unknown & 12.9 & 67.5 & 23.9 & 8.6 \\
\hline \multicolumn{5}{|l|}{ Father's smoking status $* * *$} \\
\hline Doesn't smoke & 48.7 & 85.7 & 8.1 & 6.1 \\
\hline
\end{tabular}


Table 1 continued

\begin{tabular}{|c|c|c|c|c|}
\hline & \multirow{2}{*}{$\begin{array}{l}\text { Percent distribution of } \\
\text { characteristics for full } \\
\text { sample of mothers }\end{array}$} & \multicolumn{3}{|c|}{ Cross-tabulations of smoking status by maternal characteristics (rows total to $100 \%$ ) } \\
\hline & & Non-smoker & Constant smoker & Postpartum smoker \\
\hline Does smoke & 37.5 & 54.8 & 28.7 & 16.4 \\
\hline Father info missing & 13.9 & 73.2 & 17.7 & 9.1 \\
\hline \multicolumn{5}{|c|}{ Religious attendance*** } \\
\hline $1+/$ week & 22.2 & 82.0 & 10.7 & 7.3 \\
\hline$<1 /$ week & 63.7 & 71.1 & 17.6 & 11.3 \\
\hline Never attends & 14.1 & 63.2 & 25.5 & 11.3 \\
\hline \multicolumn{5}{|c|}{ Father suggested abortion** } \\
\hline Yes & 9.8 & 66.2 & 24.0 & 9.9 \\
\hline No & 90.2 & 73.1 & 16.4 & 10.5 \\
\hline$N$ & 3,522 & 2,550 & 606 & 366 \\
\hline
\end{tabular}

Non-smokers did not smoke during pregnancy nor during the year following birth; constant smokers smoked both during pregnancy and in the year following birth, and postpartum smokers did not smoke during pregnancy but smoked in the year following birth

Source: Fragile Families and Child Wellbeing Study

** $P<0.01$; *** $P<0.001$, on the basis of $\chi^{2}$ tests, that the distribution of smoking status is random across variable categories, using the sample with complete data

missing. A non-father measure of social support is the mother's level of religious service attendance, under the assumption that frequent attendance at religious services provides mothers with social networks and psychosocial support. We distinguish between mothers who attend religious services once or more per week, mothers who attend less than once per week, and mothers who never attend.

\section{Analysis}

We begin by describing the sample characteristics and comparing the rate of constant, postpartum and nonsmoking for each category of our predictor variables. Then we use multinomial logistic regression to predict the risk of being a constant or resumer smoker relative to non-smoking for each set of predictor variables. Four models separately test the combined effects of each set of predictor variables, and a fifth model includes all variables, in order to assess the importance of each set of factors in explaining maternal smoking behavior. We used Stata/SE version 11 software (StataCorp LP, College Station, Texas) for all statistical analyses.

\section{Results}

Table 1 shows the sample distributions of the socioeconomic, healthcare, life course and health, and partner and social support measures used in this study (see column 1), as well as the bivariate distributions of smoking status by mothers' characteristics (see columns 2-4). All variables included in our analysis are significantly associated with smoking status, based on chi-square tests of equal distributions. That is, the distribution of smoking status for mothers defined by the different characteristics identified in the table is significantly different from the distribution that would be expected if smoking were independent of these characteristics. In general, the table shows that mothers with greater disadvantage-i.e., younger mothers who have less education, who live in households below the poverty level, who receive late or no prenatal care, who have public or no health insurance, who have a mental health problem, who report parenting stress, who did not breastfeed, who are unmarried, whose partners smoke, who do not attend religious services, and whose partners suggested an abortion-are more likely to be constant or postpartum smokers than mothers who fall in the more advantaged categories across these characteristics.

One exception to this general rule is race/ethnicity. Mirroring the national pattern, the highest rates of constant smoking are among the generally more advantaged nonHispanic white women. Non-Hispanic black women have lower rates of smoking than non-Hispanic white women, and rates of smoking are even lower among Hispanic women.

Table 1 also shows a second predominant pattern with several interesting exceptions. For most women, constant smoking is more prevalent than postpartum smoking. Among all mothers in the sample, for example, $17 \%$ are constant smokers, and $10 \%$ are postpartum smokers. This pattern is true for white women, black women, and regardless of education level, poverty status, prenatal care, 
health insurance coverage, age, parity, mental health, parenting stress, father's smoking status, religious attendance, and whether the father suggested an abortion.

However, the pattern of constant and postpartum smoking is different for Hispanic mothers, for mothers who breastfeed for at least 5 months, and for married mothers, among whom postpartum smoking is more common than constant smoking. About one in ten US-born MexicanAmerican and other Hispanic women are constant smokers, but $15 \%$ of these women are postpartum smokers. Foreignborn Mexican-American women have very low rates of smoking overall, but as with US-born Mexican-American and other Hispanic women, they are more likely to be postpartum than constant smokers (4 vs. 1.5\%). Among married mothers and those who breastfed for at least 5 months, about $8 \%$ are postpartum smokers, whereas only $6 \%$ of married mothers and $7 \%$ of mothers who breastfed for at least 5 months are constant smokers. Although the rate of postpartum smoking among married mothers and long-term breastfeeders is not substantially higher than the rate of constant smoking, and it is lower than the rate of postpartum smoking among unmarried and short-term or non-breastfeeders, the important point is that the pattern is different. This different pattern may point to women who are effectively "reached" by health messages regarding smoking during pregnancy, and who adjust their behavior accordingly (i.e., stop smoking) during pregnancy, but not necessarily afterwards.

A final pattern to note in Table 1 is that constant smoking varies more with socioeconomic and psychosocial factors than does postpartum smoking. For example, 9.6\% of mothers with a college education are constant smokers, compared to $23.1 \%$ of mothers with less than a high school education, a more than $100 \%$ difference. By comparison, $8.4 \%$ of mothers with a college education are postpartum smokers, compared to $12.8 \%$ of mothers with a less than high school education, a $50 \%$ difference. Similarly, the rate of constant smoking is twice as high for mothers who are below the poverty line versus $300 \%$ above the poverty line, who have public versus private insurance, and who never attend church versus attend weekly, and it is more than three times as high for mothers who are not in a romantic relationship with the infant's father (versus married) and whose infant's father smokes (versus doesn't smoke). By comparison, with the exception of fathers who smoke (in which case the difference is over $100 \%$ ), the difference in rates of postpartum smoking across these same categories are never greater than $50 \%$. This is the first piece of evidence that the profile of constant smokers is easier to identify than the profile of resumer, or postpartum, smokers.

Table 2 shows results from multinomial logistic regression equations predicting constant and postpartum smoking relative to non-smoking. In Models 1 through four we examine the effects of each separate set of variables on smoking. The associations correspond to those reported in Table 1, and in Models 1-4 they are almost all statistically significant. In Model 5, which includes all variables, poverty is no longer significantly or substantially associated with smoking. This suggests that the distribution and association of other factors-health care, life course and health, and partner and social support-with smoking status explains the higher risk of smoking among impoverished mothers.

All models show a similar pattern of stronger and more statistically significant associations for constant smoking than for postpartum smoking, the second piece of evidence that the profile of constant smokers is more easily identifiable. For example, less than high school education is associated with a four-fold increase in the risk of constant smoking relative to non-smoking, compared to a $94 \%$ increase in the risk of postpartum smoking relative to nonsmoking. In the full model, all but four measures have statistically significant associations with constant-smoking, whereas fewer than half of all measures are statistically significantly associated with postpartum smoking.

\section{Conclusions}

The results of our study suggest that among postpartum mothers, there is a much clearer risk profile for constant smokers (those who smoked during pregnancy and continued to smoke at 1 year postpartum) than there is for mothers who did not smoke during pregnancy but became postpartum smokers. Disadvantage in terms of the various psychosocial factors we measured, including measures of socioeconomic status, health care, life course and maternal health, and partner and social support, was more predictive of constant smoking than of postpartum smoking. The prevalence of constant smoking is nearly universally higher than the prevalence of postpartum smoking. In other words, post-partum smokers-mothers who abstain from smoking during pregnancy but begin or resume smoking in the postpartum period-are less common and harder to identify than mothers who smoke during and after pregnancy.

Psychosocial factors have a strong effect in predicting smoking that persists through pregnancy and the postpartum period. Mothers diagnosed with or prescribed medication for a mental health problem within the previous year are more likely to smoke during pregnancy and continue to do so after childbirth. Mothers who report high levels of parenting stress are also more likely to be constant smokers, but the relationship is explained by socioeconomic standing, health status, and access to social support. Mothers who are not married and mothers who never 


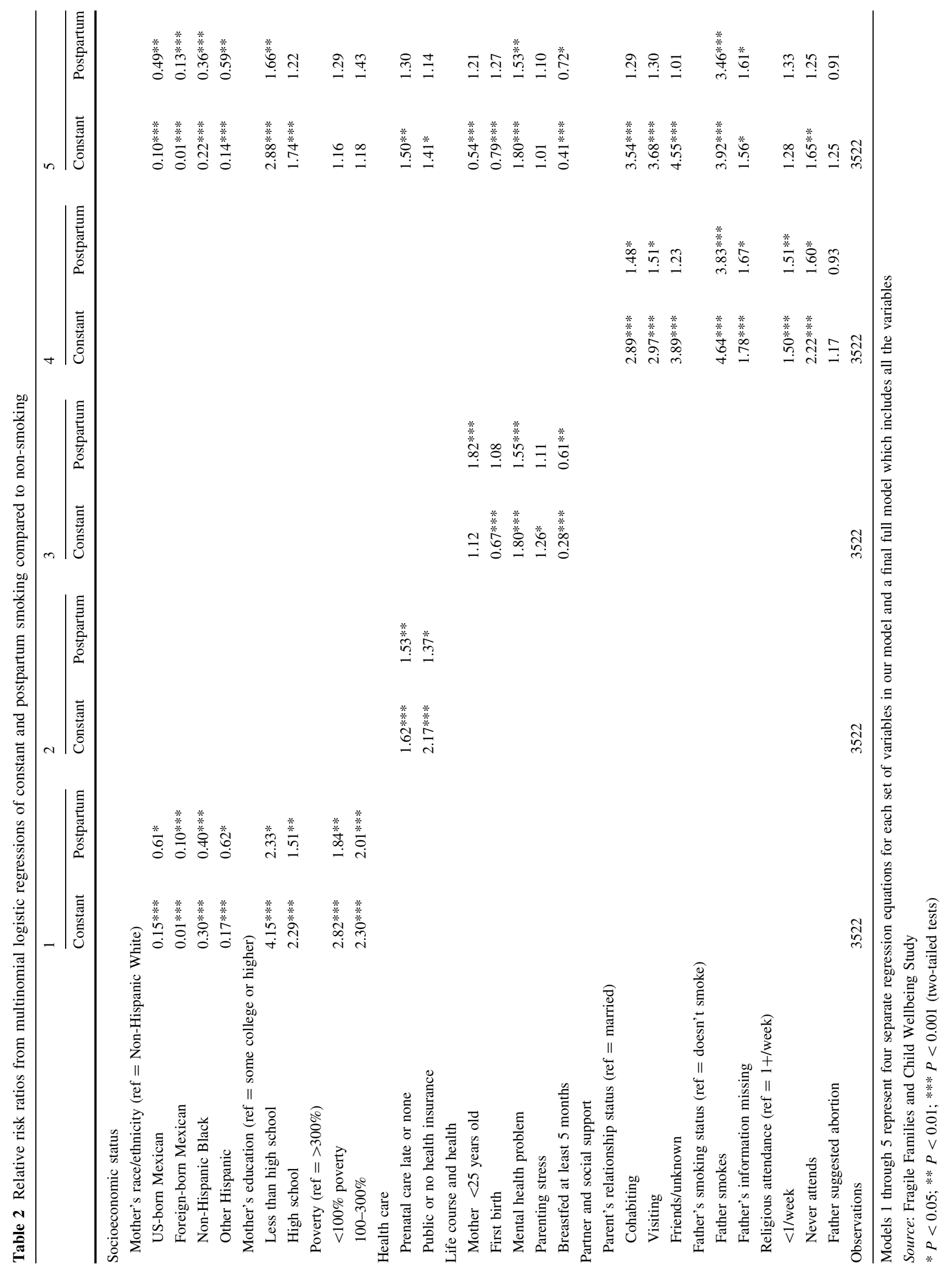


attend church, suggesting less access to social support, also have a higher likelihood of constant smoking. Finally, mothers are more likely to be constant smokers if the baby's father is also a smoker. While these factors play a role for mothers who smoke during the postpartum period but did not smoke during pregnancy, the relationship is much weaker.

Another important finding of the study concerns racial and ethnic patterns in the trajectory of maternal smoking. Overall, slightly over a quarter of mothers smoked following the birth of the child. As expected, mothers who smoked during the pregnancy also tend to continue smoking after their child's birth. Sixty percent of the postpartum smokers had smoked during their pregnancy, while $40 \%$ of the mothers who smoked during the postpartum period had not smoked during the pregnancy. Hispanic mothers, however, showed a reversal in this pattern. Hispanic mothers are more likely to be postpartum smokers than they are to be constant smokers, in contrast to White and Black women who are more likely to have smoked throughout their pregnancies. This is important because it appears that the low smoking rates among this population, which are so closely associated with healthier birth outcomes [17], may be eroded subsequent to the birth of their child.

The results can be interpreted two ways. One is that it is easier to identify or predict who will be a constant smoker and thus target interventions for these more entrenched smokers. On the other hand, it seems more difficult to identify who is at risk for postpartum smoking and thus we may miss an opportunity to intervene during this key period, while mothers are likely to be in contact with the pediatric health care system. Furthermore, Hispanic women, married women, and women who breastfeed for at least 5 months are more likely to smoke during the postpartum period in spite of having refrained from smoking during pregnancy. This suggests that the mitigating effects of prenatal care, social support, and cultural factors on smoking abstention during pregnancy may not extend as easily to the postpartum period. Healthcare providers may need to provide ongoing education and support for this particular group of women, in addition to women who engage in the more deleterious behavior of smoking both during and after pregnancy.

Although the study uncovers patterns not previously observed, we recognize some important data limitations. First, our study is based on maternal self-reports of smoking behaviors. The rate in our study of $18.8 \%$ prenatal smoking is higher than the $9-17 \%$ range based on national estimates [18], at least suggesting that the usual pattern of underreporting smoking is not a concern among this sample of predominantly unmarried, urban mothers. Maternal self-reports of smoking in the Fragile Families data used in this study are comparable to data obtained from the supplemental medical records gathered at delivery from the mothers in the Fragile Families sample [19]. However, a recent study comparing data gathered at two different times showed that maternal reports can vary significantly depending on when the question is asked. Allen and collaborators [20] compared data from birth certificates based on maternal medical records and information obtained from the mother at delivery and a self-administered questionnaire mailed approximately 2-3 months after delivery to a subsample of mothers from the birth certificates. The authors estimated that prenatal smoking may be as much as $31 \%$ higher than what is reported at delivery.

Second, we do not have information on pre-pregnancy smoking. Thus, we do not know if mothers who did not smoke during pregnancy but do so afterwards are resuming previous smoking behavior or beginning to smoke for the first time in the postpartum period. We also do not know if mothers who smoked throughout the pregnancy and beyond had a history of smoking prior to their pregnancy. Finally, because our focus is on disadvantaged, mostly unmarried mothers, comparisons with married and generally more advantaged women are necessary to determine patterns in the general population. In spite of these limitations, the study allows us to explore risk factors previously unavailable in the study of postpartum smoking.

Clearly, maternal and child health are closely intertwined, and thus a healthy pregnancy is of great concern for the health of newborns. Indeed, our findings show that the incidence of smoking among pregnant women is well above the Healthy People 2010 target of $1 \%$ or less [21]. However, the significance of the mother's health beyond the pregnancy period should not be overlooked. A number of researchers have suggested the need for maternal health care to extend beyond the traditional 6 week mark [1, 22, 23]. Such an approach would allow health care providers the opportunity to implement health promotion strategies and interventions to improve women's overall health and address continuing morbidities [22].

Acknowledgments This research was supported by the following grants: 1R01-HD-043371-01 from the National Institute of Child Health and Human Development, 5R01-HD-35301 from the National Institute of Child Health and Human Development, 5P30HD32030 from the National Institute of Child Health and Human Development, and by funding provided by: California HealthCare Foundation, The Center for Research on Religion and Urban Civil Society at the University of Pennsylvania Commonwealth Fund, Ford Foundation, Foundation for Child Development, Fund for New Jersey, William T. Grant Foundation, Healthcare Foundation of New Jersey, William and Flora Hewlett Foundation, Hogg Foundation, Christian A. Johnson Endeavor Foundation, Kronkosky Charitable Foundation, Leon Lowenstein Foundation, John D. and Catherine T. MacArthur Foundation, A.L. Mailman Family Foundation, Charles Stewart Mott Foundation, National Science Foundation, David and Lucile Packard Foundation, Public Policy Institute of California, Robert Wood 
Johnson Foundation, St. David's Hospital Foundation, St. Vincent Hospital and Health Services, and the US Department of Health and Human Services (ASPE and ACF). The authors also wish to express their gratitude to Robert A. Hummer, Professor of Sociology at the University of Texas at Austin, for his time in reviewing the manuscript and offering valuable feedback.

Open Access This article is distributed under the terms of the Creative Commons Attribution Noncommercial License which permits any noncommercial use, distribution, and reproduction in any medium, provided the original author(s) and source are credited.

\section{References}

1. Walker, L. O., \& Wilging, S. (2000). Rediscovering the "M" in "MCH": Maternal health promotion after childbirth. Journal of Obstetric, Gynecologic, and Neonatal Nursing, 29, 229-236.

2. Gaffney, K. F. (2006). Postpartum smoking relapse and becoming a mother. Journal of Nursing Scholarship, 38, 26-30.

3. Hill, S. C., \& Liang, L. (2008). Smoking in the home and children's health. Tobacco Control, 17, 32-37.

4. Ahern, J., Pickett, K. E., Selvin, S., \& Abrams, B. (2003). Preterm birth among African American and white women: A multilevel analysis of socioeconomic characteristics and cigarette smoking. Journal of Epidemiology and Community Health, 57, 606-611.

5. Bailey, B. A., \& Byrom, A. R. (2007). Factors predicting birth weight in a low-risk sample: The role of modifiable pregnancy health behaviors. Maternal and Child Health Journal, 11, 173-179.

6. Cone-Wesson, B. (2005). Prenatal alcohol and cocaine exposure: Influences on cognition, speech, language, and hearing. Journal of Communication Disorders, 38, 279-302.

7. Salihu, H. M., \& Wilson, R. E. (2007). Epidemiology of prenatal smoking and perinatal outcomes. Early Human Development, 83, 713-720.

8. Dunn, C. L., Pirie, P. L., \& Hellerstedt, W. L. (2004). Lay advice on alcohol and tobacco during pregnancy. Health Care for Women International, 25, 55-75.

9. http://oas.samhsa.gov/nsduh/2k4nsduh/2k4overview/2k4overview. pdf. Accessed 19 November 2010.

10. Johnson, J. L., Ratner, P. A., Bottorff, J. L., Hall, W., \& Dahinten, S. (2000). Preventing smoking relapse in postpartum women. Nursing Research, 49, 44-52.

11. Martin, L., McNamara, M., Milot, A., Bloch, M., Hair, E., et al. (2008). Correlates of smoking before, during, and after pregnancy. American Journal of Health Behavior, 32, 272-282.
12. Gillum, R. F. (2005). Frequency of attendance at religious services and cigarette smoking in American women and men: The Third National Health and Nutrition Examination Survey. Preventive Medicine, 41, 607-613.

13. Page, R. L., Ellison, C. G., \& Lee, J. (2009). Does religiosity affect health risk behaviors in pregnant and postpartum women? Maternal and Child Health Journal, 13, 621-632.

14. Lara, M., Gamboa, C., Kahramanian, M. I., Morales, L. S., \& Bautista, D. E. H. (2005). Acculturation and Latino health in the United States: A review of the literature and its sociopolitical context. Annual Review of Public Health, 26, 367-397.

15. Reichman, N. E., Teitler, J., Garfinkel, I., \& McLanahan, S. (2001). The Fragile Families and Child Wellbeing Study: Background, research design, and sampling issues. Children and Youth Services Review, 23, 303-326.

16. Padilla, Y. C., \& Reichman, N. E. (2001). Low birthweight: Do unwed fathers help? Children and Youth Services Review, 23, 427-452.

17. Frisbie, W. P., Forbes, D., \& Hummer, R. A. (1998). Hispanic pregnancy outcomes: Additional evidence. Social Science Quarterly, 79, 149-169.

18. Phares, T. M., Morrow, B., Lansky, A., Barfield, W. D., Prince, C. B., Marchi, K. S., et al. Surveillance for disparities in maternal health-related behaviors-Selected states, Pregnancy Risk Assessment Monitoring System (PRAMS), 2000-2001. Washington, DC: U.S. Government Printing Office; 2004. Report No. 2004-633-140/00020 Region IV.

19. Reichman, N. E., Corman, H., Noonan, K., \& Dave, D. (2009). Infant health production functions: What a difference the data make. Health Economics, 18, 761-782.

20. Allen, A. M., Dietz, P. M., Tong, V. T., England, L., \& Prince, C. B. (2008). Prenatal smoking prevalence ascertained from two population-based data sources: Birth certificates and PRAMS questionnaires, 2004. Public Health Reports, 123, 586-592.

21. U. S. Department of Health and Human Services. Healthy people 2010 (2nd ed.). Washington, DC: U.S. Government Printing Office; 2000 November. Report No.: 017-01-00547-9.

22. Misra, D. P., Guyer, B., \& Allston, A. (2003). Integrated perinatal health framework: A multiple determinants model with a life span approach. American Journal of Preventive Medicine, 25, $65-75$.

23. Schytt, E., \& Waldenstrom, U. (2007). Risk factors for poor selfrated health in women at 2 months and 1 year after childbirth. Journal of Women's Health, 16, 390-405. 